\begin{tabular}{ccc}
\hline International Journal of Engineering \& Technology, $7(4.30)(2018) 152-155$ \\
SPC \\
Website www.sciencepubco.com/index.php/IJET \\
Research paper
\end{tabular}

\title{
Cooling Effect Efficiency Prediction of Aluminum Dimples Block using DOE Technique
}

\author{
Ganesan H. N. ${ }^{1}$, Kasim M. S. ${ }^{1 *}$, Izamshah R. ${ }^{1}$, Anand T.J.S. ${ }^{1}$, Hafiz M. S. A. ${ }^{1}$, Nawi M.A.M. ${ }^{2}$, Mohamed S. B. ${ }^{3}$ \\ ${ }^{1}$ Faculty of Manufacturing Engineering, Universiti Teknikal Malaysia Melaka, \\ Hang Tuah Jaya, 76100 Durian Tunggal, Melaka, Malaysia \\ ${ }^{2}$ Faculty of Engineering Technology, Universiti Malaysia Perlis, \\ UniCITI Alam Campus, Sg. Chuchuh, 02100 Padang Besar, Perlis, Malaysia \\ ${ }^{3}$ Faculty of Innovative Design and Technology, Universiti Sultan Zainal Abidin, \\ Gong Badak Campus, Gong Badak 21300, Kuala Terengganu, Terengganu, Malaysia \\ *Corresponding authorE-mail: shahir@utem.edu.my
}

\begin{abstract}
The main aim of the present work is to study the effect of heat enhancement method on the cooling process of a spherical dimple profile. It was prominently known that introducing dimples configuration causes an enhancement in heat transfer over a surface. In this project, an experimental investigation was carried out to examine the cooling effect of the spherical dimple profile during steady laminar flow in a wind tunnel. Seventeen different sets of parameters related to dimple diameter ( $\mathrm{mm}$ ), dimple orientation (angle) and air stream velocity $(\mathrm{m} / \mathrm{s})$ were studied. The Box-Behnken of Response Surface Methodology (RSM) was used as design of experiments (DoE) tool to evaluate these parameters on cooling time. This work deals with the analysis of variance (ANOVA) in order to establish the significant effect of input parameters. The result reveals that an increase in dimple diameter and air stream velocity increase heat dissipation. The shortest cooling time of 7 minutes can be achieved when the dimple diameter is $12 \mathrm{~mm}$; the dimple orientation is $60^{\circ}$ and air flow velocity at 18 $\mathrm{m} / \mathrm{s}$. The mathematical model has been rendered where the model has been experimentally validated with the average error of $6 \%$.
\end{abstract}

Keywords: Air Stream Velocity; Cooling Time; Dimples Structure; Heat Transfer Enhancement; Response Surface Methodology.

\section{Introduction}

The conventional heat transfer augmentation technique increases either the coefficient of heat transfer or the fluid stream turbulence. The heat transfer enhancement method has gained attention in various industrial applications like aerospace industry, automotive and electronics industry. Paramount studies have been conducted on thermal performance problems. Heat transfer enhancement method can be grouped into three main ones; namely, active heat transfer improvement method, passive heat transfer improvement method and compound heat transfer improvement method [1].

The active method involves energy or power from an external source involving usage such as magnetic fields and pulse induction through plungers. The passive method requires surface modification like extended surfaces and modifications in geometric for heat transfer enhancement. The suggested passive methods for heat transfer enhancement are pin array, dimples, rib turbulators and fins [2]. The compound technique is a combination of active and passive methods to improve the heat transfer. But the passive method is more compatible as compared with other methods because the geometric modifications can be easily employed [3]. The flow separation and flow attachment region are further improved with passive method, spherical dimples as compared to other techniques [4]. Ample of efforts have been taken to develop new ideas for heat transfer improvement. Recently, dimples have pulled more attention because of the importance of the enhancement in heat transfer [5]. Many researchers have extensively conducted experimental and numerical studies for the use of dimples to enhance heat transfer.

An experimental investigation of dimple effects on drag reduction was conducted by Bearman and Harvey [6]. The study shows that dimple profiles create a boundary layer transition without the drag penalties related to sand roughness. The dimpled ball curve remains almost constant at critical Reynolds numbers. As a conclusion, dimple profile surface greatly impacts the drag reduction and laminar-turbulent transition than sand roughness. A study on spherical dimples influences on total heat transfer rate and pressure drop of flat surface of a turbulent flow was carried out in 1993. The result of the study shows that there is no change in pressure drop but the overall heat transfer rate improved to about $30 \%$ to $40 \%$ [7]. So, dimple profile gives vital effects on heat transfer rate without pressure drop. Beves et al. [8] evaluated that the application of dimple contour on a smooth surface augments the overall heat transfer rate with least pressure drop. Dimple profile not only fastened the heat transfer but it also lowers the pressure drop penalties [9].

Dimple profile increases the coefficient of heat transfer. This is because of the boundary layer of the mainstream was separated during air flow parallel to dimple block. The mainstream separated at the dimple entrance and recirculation zone is created in the upstream side of the dimples. The separated mainstream flow reattaches in the downstream side of the dimple surface and the reattached flow forms a twin vortex. [10-12]. The other benefit of dimple profile it is less costly and the overall weight of the product is lighter due to the material removal [13]. 
As compared to other methods, the dimple method proves as the most significant method in improving thermal performance. Workpiece with staggered dimple arrangements have a great heat transfer coefficient of about $26 \%$ as compared to workpiece with smooth surface. The improvement in heat transfer rate fastened the cooling process [14]. From previous studies, dimple profiled surface exhibits a greater performance in heat transfer. However, the influence of heat transfer towards cooling time has no report in the literature. In addition, the effects of dimple diameter on the heat transfer have not been reported as well. Therefore, the prime objective of this study is to observe the lower cooling time of a dimple surface with different diameters. Meanwhile, the effect of dimple arrangement and air stream velocity will be included in this work. The results of this research aim to serve the heat transfer application.

\section{Methodology}

The experimental setup for the cooling process is as shown in Figure 1. The experimental study was conducted in a subsonic wind tunnel with a steady laminar flow. Air was used as the fluid in this study and generated by an air blower at room temperature. The air stream was flowed from effuser to test section, where the workpiece was placed at the test section. The velocity of air stream was controlled by frequency inverter to ensure a steady laminar flow. The dimple profile was created on a workpiece according to the dimple parameter as detailed in Table 1 . The workpiece material that was used in this experiment was aluminum 6061 with a final dimension of $135 \times 100 \times 30 \mathrm{~mm}(\mathrm{~L} \times \mathrm{W} \times \mathrm{H})$ as shown in Figure 2. For this study, there are 9 workpieces that were used with various dimple parameters to investigate the heat dissipation. The dimple workpieces were heated over a hot plate heater at the test section of wind tunnel. Once the workpiece achieved the preferred initial temperature $\left(60^{\circ} \mathrm{C}\right)$, the air stream started to flow over the workpiece in parallel direction. As the air stream flew over, the heat energy from the dimple workpiece started to dissipate. The changes in the temperature were monitored and measured through T-type thermocouple. The workpiece was connected with 4 cables of thermocouple data logger and linked to a high-performance personal computer. The temperature drop was measured every 1 minute interval until the workpiece achieved room temperature. The temperature drop was plotted in a graph and table through PicoLog Recorder Software as shown in Figure 3. This experimental step was conducted for 9 workpieces with a total experiment number of 17 run. Analysis of variance (ANOVA) was used to evaluate the relative significance of the dimple parameters with regards to the heat dissipation.

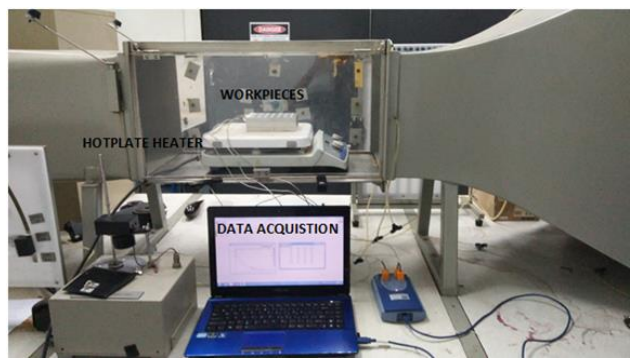

Fig. 1: Photograph of the experimental setup in a wind tunnel

Table 1: Parameters of dimple geometry

\begin{tabular}{|c|c|}
\hline Dimple Features & Measurement \\
\hline Diameter of dimple $(\mathrm{mm})$ & $14-10$ \\
\hline Orientation of dimple $\left(\Theta^{\circ}\right)$ & $90-60$ \\
\hline Dimple Density (number) & $36-32$ \\
\hline
\end{tabular}

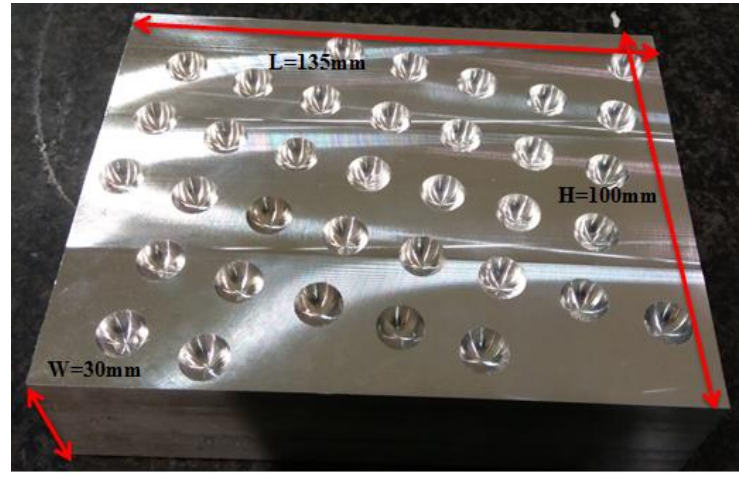

Fig. 2: Dimpled profile workpiece under study with dimension

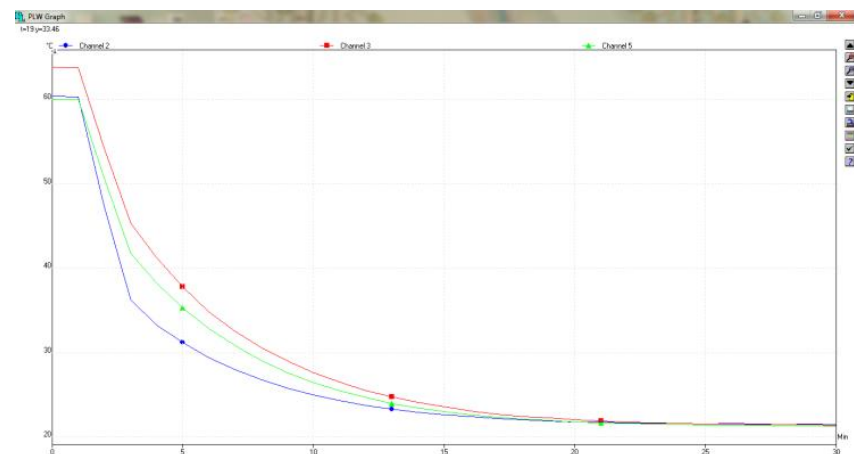

Fig. 3: Temperature drop during cooling process over time $(\mathrm{d}=14 \mathrm{~mm}$, $\Theta=75^{\circ}, \mathrm{V}=16 \mathrm{~m} / \mathrm{s}$ )

\section{Results and Discussions}

Figure 4 shows the observations of cooling time for every experiment run. Experiment no 2 of parameters d: $12 \mathrm{~mm}, \Theta: 60^{\circ}$ and V: $18 \mathrm{~m} / \mathrm{s}$ produces the lowest cooling time of dimple block, which is 7 minutes. The lower the value of the cooling time expressed, the higher the heat transfer. Experiments no 12 and no 13 present the highest cooling time of about 13 minutes. In both experiments, the diameter of dimple and velocity are the same at $10 \mathrm{~mm}$ and $17 \mathrm{~m} / \mathrm{s}$, respectively, while the dimple orientation were set at $90^{\circ}$ and $60^{\circ}$ for respective experiments no 12 and no 13. Therefore, from the observation, it can be concluded that the dimple diameter and air flow velocity are most affecting factor of the heat transfer rate. This is due to the increase in airflow velocity at the inlet, which causes the Reynolds number to be higher. When the airflow velocity increases the collision between air particles is greater, which promotes maximum vortex generation [15].

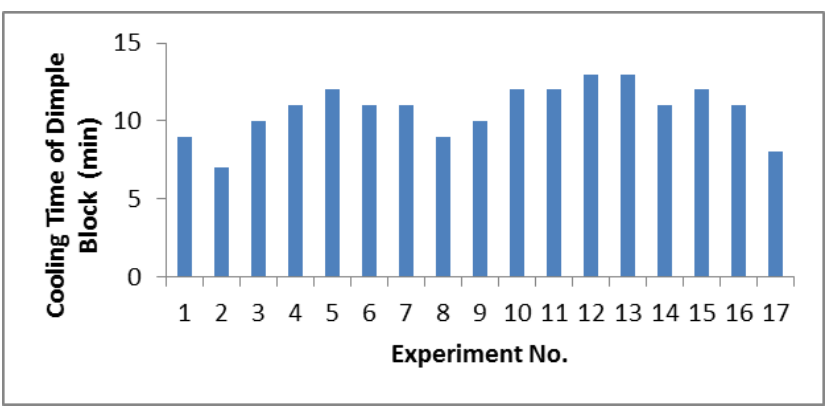

Fig. 4: Graph of cooling time throughout the experiment

In this experimental study, the performance of the dimple surface was evaluated through response surface methodology (RSM). Box Behnken design method is applied with 3 input parameters; namely, dimple diameter $(\mathrm{mm})(\mathrm{A})$, dimple orientation $\left(^{\circ}\right)(\mathrm{B})$ and air stream velocity $(\mathrm{m} / \mathrm{s})(\mathrm{C})$ over the output response as overall cooling time of a dimple workpiece. The range of input parameters is 
shown in Table 2. In RSM, minimum value denotes as -1, whereas +1 represents the maximum value of each input parameters.

Table 2: Actual and Coded values for different parameters

\begin{tabular}{|c|c|r|r|r|r|}
\hline Factor & Type & $\begin{array}{c}\text { Low } \\
\text { Actual }\end{array}$ & $\begin{array}{c}\text { High } \\
\text { Actual }\end{array}$ & $\begin{array}{c}\text { Low } \\
\text { Coded }\end{array}$ & $\begin{array}{c}\text { High } \\
\text { Coded }\end{array}$ \\
\hline A & Numeric & 10 & 14 & -1 & +1 \\
\hline B & Numeric & 60 & 90 & -1 & +1 \\
\hline C & Numeric & 16 & 18 & -1 & +1 \\
\hline
\end{tabular}

Table 3 shows the analysis of variance (ANOVA) of the cooling time of dimple block. The significant factors that affect the response can be identified when the P-value is less than 0.05. Based on ANOVA analysis done by using Design Expert software, the dimple diameter (A) and air stream velocity (C) posed significant effects than the dimple orientation (B). Even though Factor B was an insignificant factor, it cannot separate from ANOVA. This is caused by the interaction of factor B with significant factors namely $\mathrm{B} 2$ and $\mathrm{BC}$. In addition, term $\mathrm{A} 2$ and $\mathrm{C} 2$ also presented some significant effects on the cooling efficiency.

Table 3: Analysis Variance of cooling time of dimple block

\begin{tabular}{|c|c|c|c|c|c|c|}
\hline Source & $\begin{array}{c}\text { Sum of } \\
\text { Squares }\end{array}$ & DF & $\begin{array}{c}\text { Mean } \\
\text { Square }\end{array}$ & $\begin{array}{c}\mathbf{F} \\
\text { value }\end{array}$ & $\begin{array}{c}\text { Prob }> \\
\mathbf{F}\end{array}$ & \\
\hline Model & 44.08 & 9 & 4.90 & 23.64 & 0.0002 & Significant \\
\hline $\mathrm{A}$ & 6.12 & 1 & 6.12 & 29.57 & 0.00010 & Significant \\
\hline $\mathrm{B}$ & 0.13 & 1 & 0.13 & 0.60 & 0.4627 & \\
\hline $\mathrm{C}$ & 2.00 & 1 & 2.00 & 9.66 & 0.0171 & Significant \\
\hline $\mathrm{A}^{2}$ & 8.55 & 1 & 8.55 & 41.28 & 0.0004 & Significant \\
\hline $\mathrm{B}^{2}$ & 1.39 & 1 & 1.39 & 6.72 & 0.0358 & Significant \\
\hline $\mathrm{C}^{2}$ & 22.76 & 1 & 22.76 & 109.88 & $<0.0001$ & Significant \\
\hline $\mathrm{AB}$ & 0.25 & 1 & 0.25 & 1.21 & 0.3083 & \\
\hline $\mathrm{AC}$ & 0.000 & 1 & 0.000 & 0.000 & 1.0000 & \\
\hline $\mathrm{BC}$ & 4.00 & 1 & 4.00 & 19.31 & 0.0032 & Significant \\
\hline Residual & 1.45 & 7 & 0.21 & & & \\
\hline $\mathrm{R}^{2}$ & 0.9682 & & & & & \\
\hline
\end{tabular}

The prediction of the cooling time (CT) is given by Equation 1 as follows:-

$\mathrm{CT}=-517.98-9.61 \times(\mathrm{d})-0.84 \times(\Theta)+73.55 \times(\mathrm{V})+0.36 \times\left(\mathrm{d}^{2}\right)-2.55 \times 10^{-}$

${ }^{3} \times\left(\Theta^{2}\right)-2.33 \times\left(\mathrm{V}^{2}\right)+8.33 \times 10^{-3} \times(\mathrm{d}) \times(\Theta)-8.16 \times 10^{-}$

${ }^{15} \times(\mathrm{d}) \times(\mathrm{V})+0.07 \times(\mathrm{d}) \times(\mathrm{V})$

Based on the ANOVA, the R2 of the model is 0.9682, showing that the model fits the data. Figure 5 shows the comparison of mathematical model of calculated and observed data of actual experiment. The error between experimental result and statistical prediction calculation was in $1.1 \%-5.3 \%$ range. Run no. 11 shows the lowest cooling time of $6.87 \mathrm{~min}\left(\mathrm{~d}=14 \mathrm{~mm}, \Theta=60^{\circ}\right.$ and $\mathrm{V}=18 \mathrm{~m} / \mathrm{s}$ ).

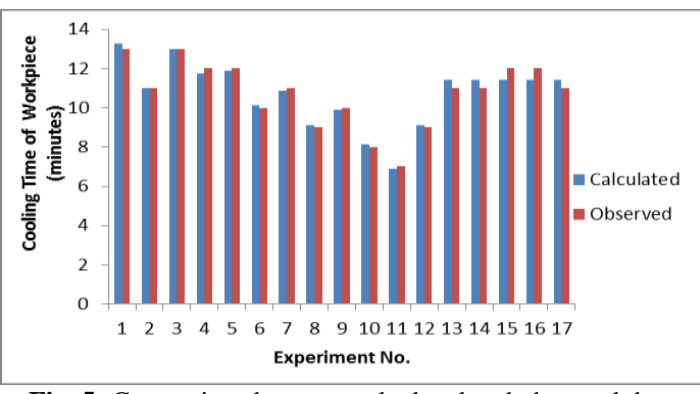

Fig. 5: Comparison between calculated and observed data

The validation of the model was done by comparing the combination of parameter which is not in data set with mathematical model The cooling process of dimple block was conducted as per selected parameters, as shown in Table 4. The output, which is the cooling time of these parameters, is 9 minutes and 11 minutes. By substituting the parameter values into the mathematical model, the predicted cooling time obtained were 9.43 minutes and 10.2 minutes. This explains that there is a difference between the predicted and the actual value. The error of both experiment sets were 4 and $7.8 \%$. The error is at an unacceptable range, which is below than $10 \%[16]$.

Table 4: Validation of prediction model
\begin{tabular}{|c|c|c|c|c|c|}
\hline $\begin{array}{c}\text { Ex- } \\
\text { per- } \\
\text { imen } \\
\text { t No. }\end{array}$ & $\begin{array}{c}\text { A: Dim- } \\
\text { ple Diam- } \\
\text { eter,D } \\
(\mathrm{mm})\end{array}$ & $\begin{array}{c}\text { B: Dimple } \\
\text { Orientation, } \\
\Theta\left({ }^{\circ}\right)\end{array}$ & $\begin{array}{c}\text { C: Air Fow } \\
\text { Velocity,V } \\
(\mathrm{m} / \mathrm{s})\end{array}$ & $\begin{array}{c}\text { Cooling } \\
\text { Time, } \\
(\mathrm{C})\end{array}$ & $\begin{array}{c}\text { Dif- } \\
\text { fer- } \\
\text { ence } \\
/ \%\end{array}$ \\
\hline 1 & 10 & 60 & 18 & 9 & 4 \\
\hline 2 & 14 & 60 & 16 & 11 & 7.8 \\
\hline
\end{tabular}

Figure 6 shows the response of cooling time at different dimple diameters. From the graph, it is clear that the higher the dimple diameter, the lower the cooling time. As the dimple diameter is getting bigger, the total surface area of a workpiece also gets bigger. Hence, the air stream was exposed to a large surface area when it flew over the dimple block. This will enhance the heat dissipation from a great air motion. Therefore, this presented the shortest time needed for a workpiece to cool down. From the graph, at one point (after $13 \mathrm{~mm}$ ), the cooling time started to increase even though the dimple diameter is bigger. This is due to the dimple radius to dimple depth ratio. As the diameter of dimple increases, the depth of dimple similarly increases. This deeper depth of dimple causes the vortex generation to take place in a longer time as compared to shallower dimple [17]. So, the dissipation of heat energy from deeper dimple is very difficult. In short, the diameter of a dimple shouldn't be too small or too big. The recommended dimple diameter range as a result from this study is $12 \mathrm{~mm}$ to $13 \mathrm{~mm}$. This is in line with findings by other researchers [18].

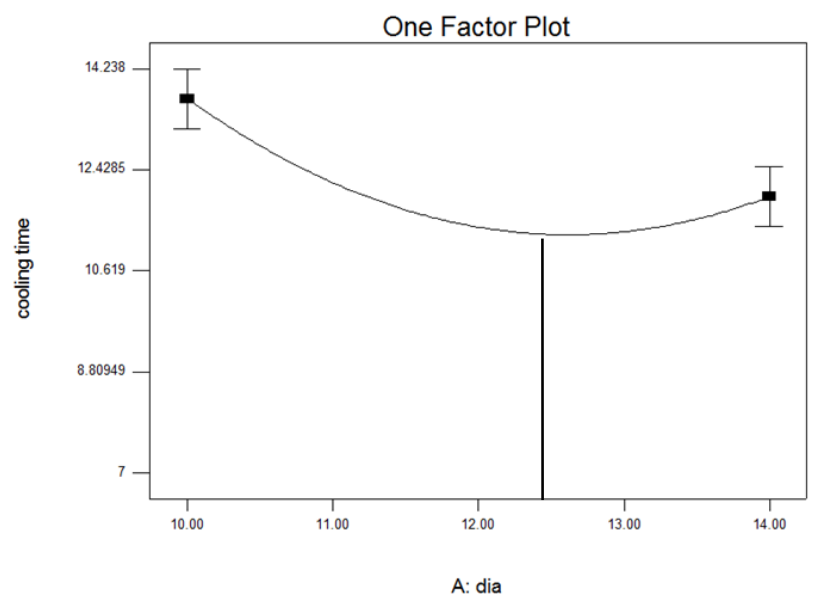

Fig. 6: Graph of dimple diameter against cooling time

The plot of the cooling time against dimple orientation is shown in Figure 7 It can be observed that the cooling time decreases when the dimple orientation in staggered arrangement $\left(60^{\circ}\right)$ as compared to inline arrangement, which is in $90^{\circ}$ orientation. However, this graph is insignificant. This study found that the shaded region $\left(\Theta=70^{\circ}-80^{\circ}\right)$ from the graph should be avoided in order to improve the heat transfer. The $75^{\circ}$ dimple orientation which falls between inline and staggered arrangement does not carry any significant effects towards heat dissipation of the overall block. Dimpled profile surface has a much better heat transfer coefficients of about $26 \%$ as compared to flat surface with staggered arrangement, whereas for inline arrangement of dimple, the heat transfer coefficients are about $25 \%$ as compared to flat surface [14]. At low angle of dimple orientation, the heat transfer coefficient yields higher values [19]. The heat dissipation will enhance when the coefficient of heat transfer is higher. Therefore, the dimple with staggered orientation $\left(60^{\circ}\right)$ dissipates heat energy faster from the dimple block as compared to the dimple with $90^{\circ}$ orientation. 


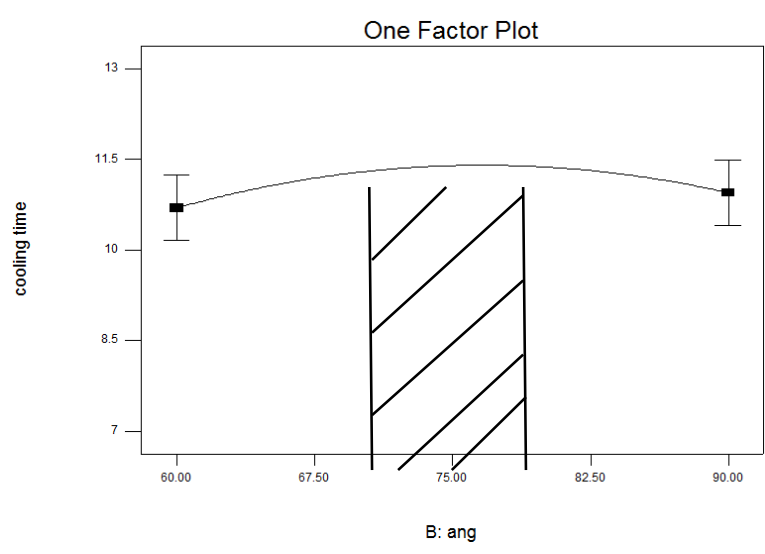

Fig. 7: Graph of dimple orientation against cooling time

The effect of air stream velocity towards cooling time is shown in Figure 8 . When the velocity of air stream increased from $16 \mathrm{~m} / \mathrm{s}$ to $17 \mathrm{~m} / \mathrm{s}$, the time taken for the workpiece to cool down also increased from 9.5 minutes to 11.5 minutes. However, the cooling time of workpiece decreased from 11.5 minutes to 8.7 minutes when the velocity of the air stream started to increase from $17 \mathrm{~m} / \mathrm{s}$ to $18 \mathrm{~m} / \mathrm{s}$. As a result, the marked area in the graph $(\mathrm{V}=16.50-$ $17.25 \mathrm{~m} / \mathrm{s}$ ) promoted poor heat transfer. Consequently, it increased the time taken of the workpiece to cool down. Therefore, the shortest cooling time obtained is at $18 \mathrm{~m} / \mathrm{s}$ velocity.

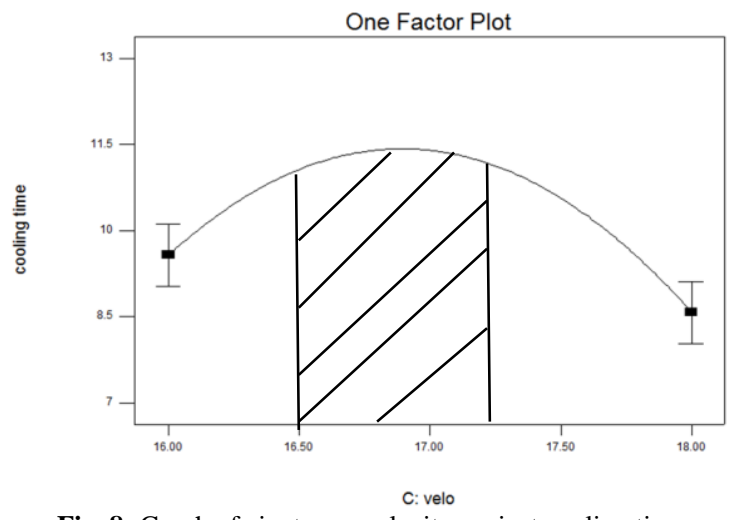

Fig. 8: Graph of air stream velocity against cooling time

\section{Conclusion}

Dimple diameter and the air stream velocity are significant factors that affect the cooling time of dimple workpiece. However, the orientation of dimple does not seem to have any significant effects on the same. The dimple diameter is the most significant factor that affects the heat dissipation of dimple block, followed by the air stream velocity. The best set of the parameters was in the case that the dimple diameter is $12 \mathrm{~mm}$, the dimple orientation is $60^{\circ}$ and the air stream velocity is $18 \mathrm{~m} / \mathrm{s}$, in order to generate a low cooling time of about 7 minutes.

\section{Acknowledgement}

Authors would like to gratefully acknowledge the Faculty of Manufacturing Engineering, Universiti Teknikal Malaysia for providing assistance to this study. This research was supported by the Universiti Teknikal Malaysia Melaka through project no. PJP/2016/FKP/HI6/S01485.

\section{References}

[1] Mahureand A \& Kriplani V (2012), Review of heat transfer enhancement techniques. International Journal of Engineerin 5, 241 249.

[2] Pisal HC \& Ranaware AA (2012), Heat transfer enhancement by using dimpled surface. Journal of Mechanical and Civil Engineering (IOSR-JMCE) 6, 07-15.

[3] Sheikholeslami M, Gorji BM \& Ganji DD (2015), Review of heat transfer enhancement methods: focus on passive methods using swirl flow devices. Renewable and Sustainable Energy Reviews 49 444-469.

[4] Lake JP, King PI \& Rivir RB (2000), Low Reynolds number loss reduction on turbine blades with dimples and V-grooves. AIAA Paper $00-738$

[5] Vorayos N, Katkhaw N, Kiatsiriroat T \& Nuntaphan A (2016), Heat transfer behavior of flat plate having spherical dimpled surfaces. Case Studies in Thermal Engineering, 8, 370-377.

[6] Bearman PW \& Harvey JK (1976), Golf Ball Aerodynamics. Aeronautical Quarterly 27, 112-122.

[7] Afanasyev VN, Chudnovsky YP, Leontiev AI \& Roganov PS (1993), Turbulent flow friction and heat transfer characteristics for spherical cavities on a flat plate. Experimental Thermal and Fluid Science, 7, 1-8

[8] Beves CC, Barber TJ \& Leonardi E (2004), An investigation of flow over two-dimensional circular cavity. In 15th Australasian Fluid Mechanics Conference, the University of Sydney, Australia, 13-17.

[9] Zhang D, Zheng L, Xie G \& Xie Y (2014), An experimental study on heat transfer enhancement of non-newtonian fluid in a rectangular channel with dimples/protrusions. Journal of Electronic Packaging 136, 021005-10.

[10] Khalatov A, Byerley A, Ochoa D \& Seong KIM (2004), Flow characteristics within and downstream of spherical and cylindrical dimple on a flat plate at low Reynolds numbers. Proceedings of ASME Turbo Expo, 1-13.

[11] Ligrani PM, Harrison JL, Mahmmod GI \& Hill ML (2001), Flow structure and local Nusselt number variations in a channel with dimples and protrusions on opposite walls. International Journal of Heat and Mass Transfer 44, 4413-4425.

[12] Griffith TS, Al-hadhrami L \& Han JC (2003), Heat transfer in rotating rectangular cooling channels $(\mathrm{AR}=4)$ with dimples. Journal of Turbomachinery 125 .

[13] Gadhave G \& Kumar P (2012), Enhancement of forced convection heat transfer over dimple surface-review. International Multidisciplinary e-Journal 1, 51-57.

[14] Katkhaw N, Vorayos A, Kiatsiriroat T, Khunatorn Y, Bunturat D \& Nuntaphan A (2014), Heat transfer behavior of flat plate having 45 ellipsoidal dimpled surfaces. Case Studies in Thermal Engineering, 2, 67-74.

[15] Alam AZMS, Islam MK, Mia MY \& Rahman KA (2014), Simulation on heat transfer enhancement in a circular tube for laminar flow with and without insert. In: Proceedings of the International Conference on Mechanical Engineering and Renewable Energy, 1 3

[16] Hills R \& Trucano T (1999), Statistical validation of engineering and scientific models: Background. Sandia National Laboratories, SAND99-1256.

[17] Burgess NK, Oliveira MM \& Ligrani PM (2003), Nusselt number behavior on deep dimpled surfaces within a channel. Journal of Heat Transfer 125, 11-18.

[18] Bi C, Tang GH \& Tao WQ (2013), Heat transfer enhancement in mini-channel heat sinks with dimples and cylindrical grooves. Applied Thermal Engineering 55, 121-132.

[19] Khan A, Bellary MZ, Ziaullah M \& Kaladgi AR (2015), An experimental study on heat transfer enhancement of flat plates using dimples. American Journal of Electrical Power and Energy Systems 4, 34-38. 